\title{
Cognitive impairment as marker of diffuse brain abnormalities in early relapsing remitting multiple sclerosis
}

\author{
M S A Deloire, E Salort, M Bonnet, Y Arimone, M Boudineau, H Amieva, B Barroso, J-C Ouallet, \\ C Pachai, E Galliaud, K G Petry, V Dousset, C Fabrigoule, B Brochet
}

J Neurol Neurosurg Psychiatry 2005;76:519-526. doi: 10.1136/jnnp.2004.045872

See end of article for authors' affiliations

Correspondence to: Professor Bruno Brochet, EA 2966 Neurobiology of Myelin Disorders Laboratory, University Victor Segalen, case 78, 146 rue Léo Saignat, 33076 Bordeaux cedex, France; bruno.brochet@ chu-bordeaux.fr

Received 17 May 2004 In revised form 22 July 2004

Accepted 4 August 2004
Objectives: To establish the frequency of cognitive impairment in a population based sample of patients with recently diagnosed relapsing-remitting multiple sclerosis (RRMS), and to determine the relation between cognitive abnormalities and the extent of macroscopic and microscopic tissue damage revealed by magnetic resonance imaging (MRI) and magnetisation transfer (MT) imaging.

Methods: 58 patients with RRMS consecutively diagnosed in the previous six months in Aquitaine and 70 healthy controls underwent a battery of neuropsychological tests. Lesion load and atrophy indices (brain parenchymal fraction and ventricular fraction) were measured on brain MRI. MT ratio (MTR) histograms were obtained from lesions, normal appearing white matter (NAWM), and normal appearing grey matter (NAGM). Gadolinium enhanced lesions were counted.

Results: 44 RRMS patients could be individually matched with healthy controls for age, sex, and education. Patients performed worse in tests of verbal and spatial memory, attention, information processing speed, inhibition, and conceptualisation. Measures of attention and information processing speed were correlated with lesion load, mean NAWM MTR, and the peak location of the NAGM MTR histogram in the patients. Multivariate regression analysis showed that lesion load and mean NAWM MTR were among the MR indices that were most significantly associated with impairment of attention and information processing speed in these early RRMS cases.

Conclusions: Cognitive impairment appears to be common in the early stages of RRMS, mainly affecting attention, information processing speed, memory, inhibition, and conceptualisation. The severity of these deficits reflects the extent of the lesions and the severity of tissue disorganisation outside lesions.
C ognition, which includes attention, language, and memory, is subserved by interconnected neural networks that contain anatomically separate channels for transferring information. ${ }^{1}$ Focal lesions or diffuse alteration of the axons can interrupt these networks. The pathological characteristics of multiple sclerosis-combining both multiple discrete lesions and diffuse axonal pathology-may explain the frequent finding of cognitive dysfunction in this disease. Various cognitive deficits mainly affecting memory and attention have been reported in up to $60 \%$ of patients. ${ }^{23}$ Although early studies suggested that the cognitive impairment is more prevalent in the later stages of the disease, ${ }^{45}$ some showed that they may be detectable at initial presentation, ${ }^{6-8}$ during the early phase, ${ }^{9-11}$ or in the presence of limited physical disability. ${ }^{12-14}$ Few studies have compared patients at the onset of their disease with appropriately matched healthy controls, and the frequency and extent of cognitive deficits during the early disease stages are still unknown.

Magnetic resonance imaging (MRI) provides an opportunity to investigate the underlying pathological basis of cognitive dysfunction in multiple sclerosis. ${ }^{15}$ Many studies focusing on classical magnetic resonance indices such as the measurement of lesion load on T2 or proton density weighted images show a weak correlation between cognitive impairment and the underlying disease process. ${ }^{16-21}$ However, the lack of pathological specificity of the lesion load limits the value of these results. The latest MRI techniques make it possible to distinguish between focal and global pathological abnormalities. In particular, the level of tissue destruction within lesions and outside them in the normal appearing parenchyma can be studied by magnetisation transfer imaging (MTI). Experimental studies and pathological correlations made on necropsy tissues in humans have validated this technique for detecting demyelination and axonal loss in vivo. ${ }^{22}{ }^{23}$ Preliminary studies using MTI have shown consistent relations between diffuse tissue destruction within the brain and cognitive impairment. ${ }^{14} 192425$ However, those studies involved samples of patients with heterogeneous types of disease and varied disease duration. To establish the relative contribution of focal lesions and remote axonal destruction to cognitive deficits in multiple sclerosis, it is necessary to correlate the cognitive impairment with MRI markers of tissue destruction at an early stage when this impairment emerges.

To evaluate the underlying pathological basis of cognitive dysfunction in early relapsing-remitting multiple sclerosis (RRMS), we recruited a sample of 58 patients at the time of diagnosis and studied their clinical and neuropsychological function together with their MRI findings. To avoid bias of age, sex, and educational level—which are known to influence the results of cognitive testing ${ }^{26}-44$ patients were individually matched to healthy controls. Moreover, to avoid

Abbreviations: BNT, Boston naming test; BRB, brief repeatable battery; EDSS, expanded disability status scale; FLAIR, fast fluid attenuated inversion-recovery; ICC, intracranial cavity; MADRS, Montgomery and Asberg depression rating scale; MI, mutual information; MSFC, multiple sclerosis functional composite; MTR, magnetisation transfer ratio; NAGM, normal appearing grey matter; NAWM, normal appearing white matter; NHPT, nine hole peg test; PASAT, paced auditory serial addition test; QOL, quality of life; RFF, Ruff figural fluency test; RRMS, relapsing-remitting multiple sclerosis; SDMT, symbol digit modalities test; SRT, selective reminding test; TWT, timed 25 foot walking test; UKNDS, United Kingdom neurological disability scale; WAIS-R, Wechsler adult intelligence scale, revised; WLG, word list generation test 
the selection bias inherent in tertiary referral populations attending specialised centres, which may have led us to overestimate the occurrence of cognitive dysfunction, this population based sample was consecutively recruited by practising neurologists from a geographically defined clinical network. This report concerns the baseline evaluation of a study planned to be longitudinal.

\section{METHODS}

\section{Patients}

To avoid referral bias inherent to tertiary specialised multiple sclerosis centre populations the present sample was composed of 58 patients with a diagnosis of RRMS of less than six months' duration made by practising neurologists from a neurological network in south western France-the Aquitaine multiple sclerosis network (AQUISEP)-and referred to the coordinating centre from November 2000 to November 2001 for the purpose of that study, without other selection criteria. According to the Declaration of Helsinki, all patients gave written informed consent before entering the study, which was approved by the local ethics committee (CCPPRB Bordeaux A). Cerebrospinal fluid (CSF) analysis and MRI were mandatory before inclusion. The diagnostic criteria of Poser $e t a^{27}$ were required for enrolment in the study. Patients with other neurological disease or known psychiatric illness, history of head trauma, or history of alcohol or drug abuse were not included. The clinical course of multiple sclerosis was classified as relapsing-remitting according to Lublin and Reingold. ${ }^{28}$ All patients underwent a standardised clinical assessment, MRI, cognitive tests, and administration of a quality of life questionnaire.

\section{Control subjects}

Control data for cognitive assessment were obtained by testing a population of 70 healthy subjects, 44 of whom could be individually matched with 44 RRMS cases for sex, age ( \pm 4 years), and educational level ( \pm 2 years). Subjects with any neurological disease or known psychiatric illness, history of head trauma, or history of alcohol or drug abuse were not included. Educational level was recorded according to the number of years of schooling. Three categories were determined: low educational level, corresponding to fewer than 12 years without obtaining any diploma; middle educational level, corresponding to 12 years plus a diploma; and high educational level, corresponding to more than 12 years.

\section{Clinical evaluation}

Neurological status and disability were assessed with a predefined standardised neurological examination including walking distance assessment by trained neurologists. Kurztke functional systems and expanded disability status scale (EDSS) scores $^{29}$ were established for all the patients by a neurologist expert in multiple sclerosis, and were derived from the source data provided by the examining neurologists. The multiple sclerosis functional composite (MSFC) was also scored. This is a clinical outcome measure that includes quantitative tests for arm/hand function (nine hole peg test (NHPT)), cognition (three second version of the paced auditory serial addition test (PASAT)), and leg function and walking (timed 25 foot walk (TWT)). To calculate the MSFC score, $z$ scores were created for NHPT, PASAT, and TWT. These $z$ scores were obtained with means and standard deviations by using data from the National Multiple Sclerosis Society task force database,,$^{30}$ which includes a wide range of patients with multiple sclerosis. The composite score was calculated using a previously published formula. ${ }^{31}$ According to published recommendations, ${ }^{31}{ }^{32}$ the inability to perform an MSFC test because of multiple sclerosis related symptoms was scored with the maximum time allowed for the NHPT
(300 seconds) and TWT (180 seconds) and with the worst possible score for PASAT (no correct answers). If patients refused to participate in a test, results were scored as missing. The Montgomery and Asberg depression rating scale (MADRS) and the five grade fatigue subscale of the United Kingdom neurological disability scale (UKNDS) (formerly the Guy's scale) were used to assess depressive and fatigue symptoms, respectively. ${ }^{33}{ }^{34}$ According to the classification proposed by Snaith et al, ${ }^{35}$ a total MADRS score of between 0 and 6 indicates absence of depression, 7-19 indicates mild depression, 20-34 indicates moderate to marked depression, and 35 or more indicates severe depression.

\section{Neuropsychological assessment}

Neuropsychological testing was carried out on the same day as MRI acquisition, by a trained neuropsychologist. It included the brief repeatable battery (BRB), which is widely used in the assessment of cognitive impairment in multiple sclerosis. ${ }^{36}$ To complete the evaluation and to study inhibition, naming, non-verbal fluency, and conceptualisation more precisely, we also used a computed battery designed by Amieva et al $^{37}$ and some additional tests. Neither patients nor controls had ever had previous neuropsychological testing.

\section{Brief repeatable battery}

The BRB included a 12 word version of the selective reminding test (SRT) which evaluates verbal memory; the 10/36 spatial recall test which evaluates short term (10/36) and long term (10/36 DR) visuospatial memory; the oral version of the symbol digit modalities test (SDMT) investigating sustained and complex attention, information processing speed, and working memory; the 60 -trial versions of the PASAT (three second and two second versions (PASAT 3s, PASAT 2s)) which evaluate sustained and complex attention, information processing speed, and working memory; and the word list generation test (WLG), assessing semantic verbal fluency. These individual tasks have been described previously in detail. ${ }^{26} 3638$

\section{Inhibitory functions}

A computerised neuropsychological battery of inhibitory functions designed by Amieva et al ${ }^{37}$ evaluates attentional skills and inhibitory processing. These include the following:

- A go-no-go task involving the execution (go trials) and the inhibition (no-go trials) of a prepared motor response. The score is the time ratio between the go-no-go task and the simple reaction time task used to assess the cognitive slowing attributable to the presence of no-go trials.

- The Stroop test, exploring attention and evaluating the difficulty in inhibiting an automatic response to word reading. The score is the ratio between the time of the interference Stroop task and the time of the colour naming task used to eliminate the effect of the general slowing of naming.

\section{Additional tests}

Additional tests included the following:

- The similarities subtest of the Wechsler adult intelligence scale-revised (WAIS-R), evaluating conceptualisation ${ }^{39}$ with a total score ranging from 0 to 28 (similarities).

- The Boston naming test (BNT), exploring naming. The total number of correct answers ranges from 0 to $28 .{ }^{40}$

- The Ruff figural fluency test (RFFl, part 1 without interference; RFF2, part 2 with interference), measuring non-verbal fluency. The score is the number of correct figures. ${ }^{41}$ 


\section{Health related quality of life assessment}

Each patient was requested to complete a self administered health related quality of life (QOL) questionnaire (SEP59) on the same day as MRI and cognitive testing. The SEP59 scale is a specific QOL multiple sclerosis scale adapted in French from the MSQOL54 and is the only validated QOL scale in French patients with multiple sclerosis. ${ }^{42}$ It covers different aspects of QOL and the 59 items are pooled in 15 different subscales. In this study, we used only the overall QOL subscale from 0 to 100, with higher scores indicating a better QOL.

\section{MRI evaluation}

MRI was undertaken at least one month from the time of a relapse or from the onset of intravenous steroid treatment.

\section{Image acquisition}

Brain MRI scans were obtained on a Philips Gyroscan ACSNT 1.5 T scanner. During a single session, the following axial brain imaging studies were done without moving the patient:

- fast fluid attenuated inversion-recovery (FLAIR) images (time of repetition (TR)/time of echo (TE)/time of inversion (TI), 11 000/140/2725 seconds);

- magnetisation transfer (MT) images using a proton density sequence (TR/TE: 37/2.3 seconds; flip angle $8^{\circ}$ ), both with and without an MT saturation pulse. The saturation pulse was an off-resonance radiofrequency pulse centred $1.5 \mathrm{kHz}$ below the water frequency with a duration of $16 \mathrm{~ms}$ and a bandwidth of $250 \mathrm{kHz}$;

- Tl weighted images (TR/TE: 450/12) before and after the administration of a standard dose $(0.1 \mathrm{mmol} / \mathrm{kg})$ of gadolinium-DTPA (Gd-DTPA).

For all sequences, 26 contiguous interleaved axial slices were acquired with $5 \mathrm{~mm}$ slices, $256 \times 256$ matrices, and $230 \times 230 \mathrm{~mm}$ fields of view. The slices were positioned to run parallel to a line joining the most inferoanterior and inferoposterior parts of the corpus callosum.

\section{Image analysis}

Image analysis was carried out in two steps. Native and preprocessed data were displayed on identical Unix workstations running image evaluation software developed by Theralys Inc (Lyon, France). An automatic computed image processing and preparation phase preceded centralised image review sessions involving three neurologists and two trained researchers who were blinded to the clinical data, during which manual corrections and validation were carried out. Pregenerated masks of intracranial cavity (ICC), ventricular and external cerebrospinal fluid (CSF), multiple sclerosis lesion load, normal appearing white matter (NAWM), and normal appearing grey matter (NAGM) were presented to the blinded readers, along with native and recorded MRI data. Each reader had to correct and validate the proposed masks.

\section{MRI volumes}

All MRI sequences were spatially registered by realigning two MRI volumes. For each patient, the Tl weighted sequence was used as a reference. All other MRI sequences from the same patient were automatically recorded in relation to the reference image. The registration algorithm used mutual information (MI) as a similarity measure in order to assess the quality of match between the two volumes. A rigid three dimensional transformation was employed. According to MI and MI derivative values, a gradient descent technique was used to modify the transformation parameters (rotations and translations) iteratively in order to maximise the MI. A multiresolution, coarse to fine strategy was adopted-that is, recording starts at low resolution and once the convergence is reached, a new iteration is initialised on the basis of the result of the previous step. This strategy increases the robustness of the registration algorithm by preventing the maximisation technique from being trapped in local maxima (by initially emphasising global, large scale image structures), before further refinement based on finer details.

\section{Intracranial cavity detection}

Segmentation of intracranial volumes was undertaken in a two step process. First, masks of intracranial cavity (ICC) were automatically generated by a three dimensional segmentation algorithm applied on proton density weighted images. This segmentation algorithm integrates a spatial "regularisation" component based on a Markov random field model. The algorithm segments volumes into several "classes" representing the various anatomical tissues (ICC here), and can reach a given number of classes by successive iterations. The signal intensity is represented by a Bayesian model, the parameters of which are measured by an estimation-maximisation iterative process. The parameters are extracted from the neighbourhood of variable size to allow the algorithm to be adaptive. The neighbourhood model takes into account voxel anisotropy using the spacing of MRI data $(0.8984 \times 0.8984 \times 5 \mathrm{~mm})$. Two levels of neighbourhood are used-level 1: neighbourhoods of size $22 \times 22$ pixels $\times 5$ axial slices; level 2: neighbourhoods of size $11 \times 11$ pixels $\times 3$ axial slices. Proton density weighting images were segmented into three classes by the segmentation algorithm. The masks obtained were then postprocessed to retain only the three dimensional object representing the ICC. This posttreatment, which consists of the application of a three dimensional connectivity algorithm in which the most voluminous three dimensional object is retained, eliminates extrameningeal structures with high signal intensities.

\section{Lesion load quantification}

Hyperintense lesions were analysed on FLAIR scans. All FLAIR axial slices from the base of the cerebellum to the vertex were analysed. A mask of multiple sclerosis lesions was generated from FLAIR images after application of the ICC mask by applying a threshold on signal intensity derived from the signal intensities histogram. Two trained neurologists using drawing tools then validated each discrete lesion and corrected its delimitation if necessary.

\section{External and ventricular CSF detection}

Using the ICC masks and the MTR maps we applied a thresholding technique to detect external (extraparenchymal) and ventricular CSF. This was possible because the MTR value of water is $0 \%$. The corresponding masks were used to compute brain atrophy parameters such as brain parenchymal fraction and ventricular fraction. A 33\% threshold on MTR values was systematically used to generate the initial CSF mask. These pregenerated CSF masks were further validated. The distinction between external CSF and ventricular CSF was made by a semiautomatic technique. The lower anatomical level of the ventricular CSF was manually defined by the proofreaders and a three dimensional connectivity technique allowed ventricular and external CSF masks to be distinguished.

\section{NAWM and NAGM detection}

The normal appearing white and grey matter masks were generated from $\mathrm{Tl}$ weighted images using the same segmentation algorithm as for ICC detection. Tl weighted images were initially masked by using the validated ICC contours in order to eliminate extrameningeal structures and improve the classification of voxels belonging to the brain parenchyma and the CSF. Masked Tl weighted images were 
segmented into four classes by the automatic algorithm. These classes corresponded to the white matter, the grey matter, the CSF, and background. Previously generated masks were automatically transferred to the co-registered $\mathrm{Tl}$ images and then nulled to eliminate lesions and CSF before validation.

\section{MRI parameters}

The following parameters were taken into consideration in the analysis:

Lesion volume (lesion load, $\mathrm{cm}^{3}$ ), calculated from lesion masks.

Atrophy marker measurements: brain parenchymal fraction and ventricular fraction were measured. Brain parenchymal fraction is defined as the ratio of brain parenchyma to the intracranial volume (total volume contained within the brain surface contour), and ventricular fraction is defined as the ratio of ventricular volume to the intracranial volume. ${ }^{43} 44$ Ventricular measurement included all four ventricles.

MTR measurements: MTR maps were constructed by calculating the MTR on a pixel by pixel basis from the MTR signal intensity decay according to the formula:

$$
\mathrm{MTR}=[(\mathrm{Mo}-\mathrm{Ms}) / \mathrm{Mo}] \times 100
$$

where Ms represents the signal intensity of pixels with saturation and Mo without saturation..$^{45}$ Normalised histograms of MTR maps were computed after removal of the extracerebral structures and CSF by application of the corresponding masks. For each patient, the average MTR value was computed over the lesion masks from FLAIR. To obtain the MTR histograms of NAWM and NAGM, lesion masks from FLAIR scans were automatically transferred to the co-registered MTR maps and then nulled. For all histograms (lesions, NAWM, and NAGM), the average MTR values were calculated, as well as the heights (that is, the proportion of pixels at the most common MTR) and locations (the most common MTR) of the histogram peaks. Normalisation was carried out using the total number of voxels in each region (mask) of interest: lesions, NAWM, and NAGM. ${ }^{19}$

Number of Gd-DTPA enhancing lesions: Tl Gd-DTPA enhancing lesions were identified by agreement between two experienced neurologists on $\mathrm{Tl}$ weighted images acquired after Gd-DTPA infusion. The software automatically calculated the number of Tl Gd-DTPA enhancing lesions detected.

\section{Statistical analysis}

The SAS version 8.2 for Windows was used for all statistical analysis. Individual matching between multiple sclerosis patients and healthy controls was verified using $\chi^{2}$ test for categorical variables (sex and low/middle/high educational levels) and the paired Student $t$ test for age. The difference in cognitive performance between RRMS patients $(n=44)$ and individually matched controls was evaluated by using the paired Student $t$ test. Comparisons between non-paired RRMS $(n=14)$ and paired RRMS patients $(n=44)$ were determined by Student's $t$ test for all cognitive scores. The 5th centile of the control performance on all neuropsychological tests was taken as the cut off point for calculating the number of failed tests for all RRMS patients $(n=58)$. The 5 th centile was used because there was no significant difference in age and educational level between 44 paired RRMS patients and 14 non-paired RRMS patients ( $\chi^{2}$ test).

The univariate correlation between the low cognitive scores, MRI derived metrics, and clinical variables was assessed by using the Pearson correlation coefficient for all RRMS patients $(n=58)$. When calculating the statistical significance of the univariate correlation, no correction for multiple comparisons was made owing to the exploratory nature of this study and in order to minimise the risk of type II errors. Only correlations of at least moderate strength (that is, with an $r$ value $\geqslant 0.30$ ) and a significance level of $\mathrm{p}<0.05$ are reported.

To assess factors independently associated with abnormal cognitive scores, we carried out multivariate linear regression using lesion load, brain parenchymal fraction, ventricular fraction, lesion MTR, NAWM-MTR and NAGM-MTR histogram derived metrics as explanatory variables. Demographic variables (age, educational level, and sex) were forced into the model as confounding variables. Confounders were identified with regard to their effect on the $\beta$ coefficient and the significance of $\mathrm{p}$ value when added to the regression analysis. For each multivariate model, factors with a conservative significance level of $\mathrm{p}<0.25$ on univariate analysis were then included in a multiple linear regression model. Factors not significant at the 0.05 level were removed from the model by backward elimination.

\section{RESULTS}

The demographic and clinical data on the RRMS patients are summarised in table 1. No patient was diagnosed as severely depressed by MADRS (score >34); $1.8 \%$ of RRMS patients were diagnosed as moderately to markedly depressed (score 20-34), 26\% had mild depressive symptoms (score 7-19); and $72.2 \%$ did not display any significant depressive symptoms ( score $<7$ ).

\section{Neuropsychological results}

The performance of the 44 RRMS cases matched individually to controls according to age, sex, and educational level was similar to that of the non-matched RRMS patient group $(\mathrm{n}=14)$.

Significant differences between matched RRMS patients and matched controls $(n=44)$ were found for short term verbal memory, long term storage, and consistent long term retrieval, for SRT (SRT LTS and SRT CLTR), and SRT-delayed recall (SRT-DR), for delayed recall of visuospatial memory (10/36 DR), for all tests of attentional and information processing speed functions (SDMT, PASAT3s, and PASAT2s), for conceptualisation on the similarities subtest of the WAIS-R, and for inhibitory processing at the Stroop test, but not in the go-no-go paradigm. The data are presented in table 2. MADRS scores did not affect the significance of the results.

If cognitive impairment is defined by two or more tests scores in the lowest centiles (below the 5th), $45 \%$ of the RRMS patients $(n=44)$ fulfilled this definition; $86 \%$ of the patients scored below the 5th centile for at least one test score, and only $13 \%$ of patients had no test under the 5 th centile. Concerning memory tests, 39\% of the RRMS patients scored below the 5th centile of the performances of the controls for at least one memory variable; $57 \%$ of the RR patients scored below the 5th centile of the controls for at least one attentional task (SDMT, PASAT 3 and 2 s), and $43 \%$ for at least one inhibition task. The proportions of patients scoring less than this cut off point for each cognitive test are presented in table 2. The highest percentage was for the SDMT $(48 \%$ of patients had low scores), followed by the similarities test $(20 \%)$.

\section{Association with demographic and clinical variables}

There was no significant correlation between performance on cognitive tests and the quantity of depressive symptoms according to the MADRS. In addition, no significant correlation was found between cognitive performances and the fatigue score. EDSS was only weakly correlated with SDMT score $(p<0.01, r=-0.38)$. Among the two motor 
Table 1 Demographic and clinical characteristics of the 58 patients with relapsing-remitting multiple sclerosis

$\begin{array}{ll}\begin{array}{l}\text { Demographic variables } \\ \text { Sex (male/female) }\end{array} & 14 / 44 \\ \text { Mean age (years) } & 37.34(9.17) \\ \text { Educational level* } & 28 / 18 / 12 \\ & \\ \text { Clinical variables } & \\ \text { Disease duration (months) } & 24.33(26.49) \\ \text { Time since diagnosis (months) } & 3.21(3.15) \\ \text { EDSS } & 2.0(0.0 \text { to } 6.5) \\ \text { TWT (seconds) } & 9.18(6.93) \\ \text { NHPT (seconds) } & 22.17(5.86) \\ \text { MSFC } & 0.064(0.678) \\ \text { MADRS† } & 3(0 \text { to } 21) \\ \text { Fatigue score } & 10 \text { to } 5) \\ \text { Overall QOLt } & 68.97(18.07) \\ & \\ \text { Cognitive scores } & \\ \text { SRT LTS } & \\ \text { SRT CLTR } & 51.83(12.98) \\ \text { SRT-DR } & 40.69(16.20) \\ \text { 10/36 } & 9.91(2.23) \\ \text { 10/36-DR } & 20.53(4.81) \\ \text { SDMT } & 6.76(2.40) \\ \text { PASAT 3 s } & 41.19(12.49) \\ \text { PASAT 2 s } & 42.43(13.57) \\ \text { WLG } & 32.70(13.35) \\ \text { Similarities } & 23.88(6.34) \\ \text { BNT } & 17.19(5.07) \\ \text { RFF1 } & 23.31(3.26) \\ \text { RFF2 } & 11.88(5.04) \\ \text { Go-no-go } & 10.50(6.87) \\ \text { Stroop test } & 1.07(0.14) \\ & 1.93(1.13)\end{array}$

All scores are expressed as mean (SD) except for EDSS, MADRS, and fatigue scores, which are median (range).

*Number of patients with low/middle/high educational level (see methods).

$\lceil n=57 ; \ddagger n=56$ evaluated patients.

BNT, Boston naming test; CLTR, consistent long term retrieval; DR, delayed recall; EDSS, expanded disability status scale; LTS, long term storage; MADRS, Montgomery and Asberg depression rating scale; MSFC, multiple sclerosis functional composite; NHPT, nine hole peg test; PASAT, paced auditory serial addition test; QOL, quality of life; RFF, Ruff figural fluency test; SDMT, symbol digit modalities test; SRT, selective reminding test; TWT, timed 25 foot walking test; WLG, word list generation test; 10/36, spatial recall test evaluating short term visuospatial memory; 10/36-DR, spatial recall test evaluating long term visuospatial memory.

variables of the MSFC, the TWT $z$ score was not correlated with cognitive scores, although the NHPT $z$ score was significantly correlated with several tasks, namely SDMT $(\mathrm{p}<0.0001, r=0.53)$, PASAT $3 \mathrm{~s}$ correct answers $(\mathrm{p}<0.01$, $r=0.40)$, and PASAT 2 s correct answers $(\mathrm{p}<0.05, r=0.31)$. The overall health related QOL score derived from the SEP 59 scale was significantly correlated with SDMT mean score $(\mathrm{p}<0.01, r=0.39)$ and PASAT 3 s correct answers $(\mathrm{p}<0.05$, $r=0.34$ ), but not with other cognitive performance.

\section{Correlation with MRI parameters \\ Lesion variables}

No correlation was observed between results on memory tasks (SRT, 10/36) and lesion variables. On the other hand, lesion load was significantly correlated with attentional test scores, SDMT correct answers $(\mathrm{p}<0.0001, r=-0.51)$, and PASAT 3s correct answers $(\mathrm{p}<0.05, r=-0.34)$. No correlation was observed between inhibition tasks or similarities subtest score and lesion load. No correlation was found between mean lesion MTR and other metrics of MTR histograms of lesion masks, reflecting the severity of lesions, and any cognitive performance. No correlation was found between the number of Gd-DTPA enhanced lesions and any cognitive scores.

\section{Atrophy and cerebral volume}

Volume measurements (brain parenchymal fraction, ventricular fraction) were not correlated with cognitive scores.

\section{Parenchyma parameters}

MTR histogram metrics were used to assess tissue destruction outside visible lesions in the white and grey matter. NAWM mean MTR was significantly correlated with PASAT 3s correct answers $(\mathrm{p}<0.01, r=0.34)$. Peak location of NAGM (the MTR value most represented in the NAGM) was correlated with SDMT scores $(\mathrm{p}<0.01, r=0.38)$.

\section{Multivariate regression analysis}

Using a multivariate regression model adjusting on demographic data (age, sex, and educational level) we studied the independent association of the explanatory variables listed in the method section with low cognitive scores of RRMS patients on the following tests: SRT-LTS, SRT-CLTR, SRT-DR, 10/36-DR, SDMT, PASAT 3 and 2s, similarities subtest, and Stroop test. The model showed that lesion load remained significantly associated with $\operatorname{SDMT}(\beta=-2.8, \mathrm{p}<0.0001)$ and with similarities subtest performances $(\beta=-0.55$, $\mathrm{p}<0.05)$. Average NAWM MTR was strictly associated with the two versions of the PASAT test (PASAT 3s $\beta=6.84$, $\mathrm{p}<0.01$; PASAT 2s $\beta=4.99, \mathrm{p}<0.05)$. Average lesion MTR was significantly associated only with $10 / 36$ DR $(\beta=0.33$, $\mathrm{p}<0.05$ ) (table 3 ). The other MRI variables tested were all removed from the model.

\section{DISCUSSION}

\section{Cognitive impairment in early multiple sclerosis}

This population based sample of RRMS patients recruited near the time of diagnosis showed that they had significant cognitive deficits compared with healthy subjects individually matched for age, sex, and educational level. Their main altered functions were attention, working memory, and information processing speed (PASAT and SDMT), inhibition (Stroop), short term and delayed verbal memory (SRT), visual memory (10/36-DR), and conceptualisation (similarities). On the other hand, verbal and non-verbal fluency and naming appeared to be unaffected at this stage. Overall, $44.8 \%$ of the RRMS patients $(n=58)$ performed at less than the 5 th centile of control subjects on two or more tests. Up to $80 \%$ of RRMS patients performed less well than the 5th centile of normal subjects on at least one test. These poor cognitive performances cannot be explained by the quantity of depressive symptoms as measured by the MADRS, even though such symptoms might occur in this difficult period following disclosure of a diagnosis like multiple sclerosis.

These findings confirm and extend previous observations on cognitive performance in early multiple sclerosis. The cognitive dysfunction described in the first controlled studies carried out at the early stage of the disease mainly concerned visual or verbal memory and abstract reasoning. ${ }^{10}{ }^{11}$ More recent but uncontrolled studies in early multiple sclerosis have described significant impairment on PASAT and SDMT, ${ }^{813}$ while a later study of 67 patients with probable multiple sclerosis showed a high rate of abnormalities on the WLG test of verbal fluency. In our study, however, WLG performance did not differ significantly between patients and controls matched for educational level.

The cognitive dysfunction shown by our population of RRMS patients with a recent onset of disease concerned essentially the same functions as are commonly observed in patients with advanced multiple sclerosis, ${ }^{46}$ particularly with respect to attentional deficits, memory deficits, and conceptualisation. However deficiencies are probably less severe at this stage and may not have a significant impact on daily life. For example, the overall health related QOL score was 
Table 2 Neuropsychological performances of 44 patients with relapsing-remitting multiple sclerosis matched individually to 44 controls according to age, sex, and educational level

\begin{tabular}{llll}
\hline Neuropsychological tests & \% RRMSt & RRMS & Controls \\
\hline Age (years) & & $38.20(9.48)$ & $37.81(10.80)$ \\
Sex (M/F) & & $14 / 30$ & $14 / 30$ \\
Educational level & $19 / 13 / 12$ & $19 / 9 / 16$ \\
SRT LTS & 25 & $51.25(12.25)^{*}$ & $56.61(9.00)$ \\
SRT CLTR & 30 & $40.86(16.34)^{*}$ & $47.73(11.32)$ \\
SRT-DR & 18 & $9.88(2.17)^{* *}$ & $10.93(1.40)$ \\
10/36 & 11 & $20.11(4.74)$ & $21.98(4.97)$ \\
10/36-DR & 16 & $6.73(2.38)^{* *}$ & $8.20(1.77)$ \\
SDMT & 48 & $41.39(11.71)^{\star * * *}$ & $56.82(9.46)$ \\
PASAT 3s & 23 & $41.73(14.52)^{\star *}$ & $47.73(9.14)$ \\
PASAT 2 s & 39 & $31.57(14.30)^{\star * *}$ & $40.66(8.86)$ \\
WLG & 0 & $24.14(6.39)$ & $26.32(6.96)$ \\
Similarities & 20 & $17.59(4.94)^{* * * *}$ & $21.09(3.52)$ \\
BNT & 0 & $23.77(2.73)$ & $24.34(2.52)$ \\
RFF1 & 0 & $11.61(4.83)$ & $13.48(5.64)$ \\
RFF2 & 0 & $10.45(7.02)$ & $11.82(6.42)$ \\
Go-no-go & 7 & $1.07(0.13)$ & $1.11(0.12)$ \\
Stroop test & 36 & $1.87(0.90)^{* * *}$ & $1.34(0.29)$ \\
\hline
\end{tabular}

For all tests, the results are expressed as mean (SD) number of correct answers except for go-no-go (ratio sequential reaction time/simple reaction time) and Stroop test (ratio time to the interference Stroop task/time to the colour naming task).

tPercentage of patients scoring below the 5th centiles of paired healthy control scores for each cognitive test $(n=44)$.

$\ddagger$ Number of patients and healthy controls, respectively, with low/middle/high educational levels.

Probability ( $p$ ) values relate to comparison between paired RRMS patients and controls determined by the paired Student $t$ test for all variables except for sex and educational level $\left(\chi^{2}\right.$ test $)$ : ${ }^{*} p<0.05$; ${ }^{* *} p<0.01$; ${ }^{* * *} p<0.001$; ${ }^{* * * *} p<0.0001 ; p$ values over 0.05 were considered as non-significant.

BNT, Boston naming test; CLTR, consistent long term retrieval; DR, delayed recall; LTS, long term storage; PASAT, paced auditory serial addition test; RFF, Ruff figural fluency test; RRMS, relapsing-remitting multiple sclerosis; SDMT, symbol digit modalities test; SRT, selective reminding test; WLG, word list generation test; 10/36, spatial recall test evaluating short term visuospatial memory; 10/36-DR, spatial recall test evaluating long term visuospatial memory.

not correlated with most of the cognitive scores, except for attention and information processing speed (SDMT and PASAT 3s). The wide range of abnormalities observed with the BRB tests in this early phase of multiple sclerosis is reminiscent of those found in several other studies using the same battery in different multiple sclerosis populations. For example, two recent studies reported very low performance on sustained attention and verbal memory in patients with longer disease duration and with greater disability than our patients. ${ }^{47}{ }^{48}$ Among our patients, 36\% were impaired on the Stroop test, showing a partial breakdown in inhibitory

Table 3 Multivariate linear regression model $(n=58)$

\begin{tabular}{lll}
\hline Dependent variable & Explanatory variable & $\boldsymbol{\beta}$ \\
\hline SRT-LTS & - & \\
SRT CLTR & - & \\
SRT DR & - & 0.33 \\
10/36-DR & Average lesion MTR & \\
$R^{2}$ model $=0.15$ & Lesion load $\left(10 \mathrm{~cm}^{3}\right)^{* * * *}$ & -2.8 \\
SDMT & & \\
$R^{2}$ model $=0.49$ & Average NAWM MTR & 6.84 \\
PASAT 3 s & & \\
$R^{2}$ model $=0.25$ & Average NAWM MTR* & 4.99 \\
PASAT 2 s & Lesion load $\left(10 \mathrm{~cm}^{3}\right)^{*}$ & -0.55 \\
$R^{2}$ model $=0.23$ & & \\
Similarities & & \\
$R^{2}$ model $=0.27$ & - & \\
Stroop &
\end{tabular}

See text for details of multivariate analysis procedure. ${ }^{*}=p<0.05 ;{ }^{* *}=p<0.01 ;{ }^{* * *}=p<0.001,{ }^{* * * *}=p<0.0001$. CLTR, consistent long term retrieval; DR, delayed recall; LTS, long term storage; MTR, magnetisation transfer ratio; NAWM, normal appearing white matter; PASAT, paced auditory serial addition test; SDMT, symbol digit modalities test; SRT, selective reminding test; 10/36-DR, spatial recall test evaluating long term visuospatial memory. processes and confirming previous findings in patients with multiple sclerosis at various stages. ${ }^{13} 49$ Finally, our patients scored poorly on the similarities subtest, a test for conceptualisation, thereby confirming previous findings in various multiple sclerosis populations ${ }^{50-52}$ including patients at the early stages. ${ }^{10}$

\section{Neurobiological correlates of cognitive dysfunction}

In general, lesion load and normal appearing parenchymal integrity were significantly correlated with scores on two cognitive tests exploring attentional and information processing speed capacities. For example, lesion load was significantly correlated with SDMT and PASAT, the association between SDMT and lesion load remaining significant in the final multivariate model. Lesions may contribute to cognitive dysfunction by interrupting the conduction pathways between cortical areas. However, lesion load as measured on T2 or FLAIR indicates the extent of heterogeneous brain abnormalities, ranging from oedema and inflammation to severe demyelination and axonal loss, but it provides little information about the pathological substrates of multiple sclerosis lesions and their potential role in the low cognitive scores. ${ }^{23}$ To explore the meaningfulness of this association, we used MTI to examine the relation between the severity of tissue destruction within lesions and cognitive performance, as experimental and pathological studies have established that an MTR decrease within lesions reflects the severity of tissue destruction..$^{23}$ No univariate correlation was observed between cognitive scores and MTR histogram metrics of lesion masks. However, in the multivariate model adjusting for age, sex, and educational level, an association appeared between delayed recall of visuospatial memory score and average lesion MTR, suggesting that destruction severity within lesions can affect visuospatial 
memory dysfunction at this stage. Previous studies have shown that populations of multiple sclerosis patients with cognitive impairment present more severe lesions. ${ }^{19} 25$ However, these studies were carried out on multiple sclerosis patients with various disease durations and heterogeneous clinical forms, suggesting that the severity of the tissue destruction within lesions contributes more to the impairment at a later stage, at least indirectly by contributing to axonal disruption.

Axonal degeneration remote to lesions has been described at early stages of multiple sclerosis..$^{53}$ To examine the role of diffuse axonal loss in cognitive dysfunction in the early stages of the disease, we used MTI to study normal appearing brain tissue in our population and found significant correlations between the mean MTR of the NAWM and PASAT 3s performance. In the final multivariate model, the association between deficits observed on the PASAT $3 \mathrm{~s}$ and diffuse destruction of NAWM remained significant and was also significant on PASAT 2s. This association suggests that axonal degeneration of fibres within the intercortical networks might already be contributing to cognitive impairment at the onset of the disease, which has not been considered until now. Several previous studies have shown a correlation between mean NAWM MTR and cognitive function at later stages of the disease. ${ }^{141925}$ Rovaris et al found that patients with cognitive impairment had significantly increased lesion load on proton density weighted images, with decreased lesion MTR and total brain mean MTR, than patients without cognitive impairment in 30 subjects with a variable clinical course and with disease durations ranging from two to 23 years. ${ }^{19}$ Similar results were found in another study where tissue destruction was correlated with cognitive impairment in 19 patients with relapsing-remitting or secondary progressive multiple sclerosis with a mean disease duration of 15 years. ${ }^{25}$ In that study, logistic regression analysis showed that mean MTR of normal appearing brain tissue was more strongly associated with cognitive impairment than with the extent of $\mathrm{T} 2$ visible lesions and their severity. Our results also suggest that axonal destruction within white matter may play a critical role in attention and information processing speed by affecting intercortical networks.

Cortical lesions have recently been reassessed in multiple sclerosis, and a role in cognitive dysfunction has been suggested. Lazeron et al studied cortical and juxtacortical lesions by FLAIR in 39 patients and found a higher lesion load in these areas in patients with cognitive impairment detected by the BRB ${ }^{54}$ Rovaris et al observed reduced MTR values in cortical/subcortical regions in 16 patients with long disease duration and cognitive impairment compared with six without. ${ }^{24}$ In our early RRMS patients, only the peak location (the most frequent value) of MTR histograms in the grey matter was correlated with SDMT scores in univariate analysis, but this association did not remain in final multivariate model after adjusting for demographic data.

Brain atrophy is known to occur early in the course of multiple sclerosis and is considered to be a global marker of tissue destruction. ${ }^{55}$ At any given time, the extent of atrophy is thought to reflect the net effect of the irreversible tissue damage that has occurred so far. However, measurements of total brain volume or whole atrophy do not appear to be sensitive enough to show any correlation with cognitive performances at this early stage. Indeed, experimental studies suggest that the development of atrophy is probably delayed until after the appearance of lesions and diffuse axonal loss. ${ }^{56}$ Work addressing the relation between atrophy and disability has produced varying results, ${ }^{57-62}$ as has that attempting to correlate neuropsychological testing with atrophy. ${ }^{21}{ }^{63-65}$ This heterogeneity of observations could reflect the use of different methods of evaluation.

\section{Clinical correlations}

EDSS was only moderately correlated with the results of one cognitive test of attention (SDMT), confirming the lack of correlation of this variable with cognitive function that has been found in many studies. ${ }^{46}$ Underestimation of cognitive decline by EDSS may explain this poor correlation. ${ }^{3031}$ The NHPT, one of the motor variables of MSFC, correlated significantly with attentional and information processing speed tests (SDMT, PASAT). NHPT may require attention or could itself be dependent on processing speed.

\section{Conclusions}

Cognitive impairment is common in the early stages of multiple sclerosis, mainly affecting attention, working memory, and information processing speed, but also memory, inhibition, and conceptualisation. Poor performance is common but remains subtle and does not significantly affect the quality of life at this stage. However, our findings show that these deficits reflect the destruction both within and outside lesions, and that they may therefore be considered as a severity marker in the early stages of multiple sclerosis.

\section{ACKNOWLEDGEMENTS}

This work was made possible by the contribution of neurologists from the AQUISEP network, a clinical network of neurologists of Aquitaine and Charente devoted to multiple sclerosis care in this area. The following neurologists particularly contributed to the study: Drs Artaud-Uriot, Aupy, Bastard, Bredin, Brohée, Daviaud, Delabrousse-Mayoux, Desbordes, Devoize, Fouillet, Gayou-Joyeux, Hardy, Hegbé, Hermosilla, Laporte, Larribau, Lebrun-Grandie, Louvet, Massal, Maupetit, Pautrizel, Pin, Prat, Saudreau, Schuermans, Vincent, and Zerbib. The study is supported by grants from Association pour la Recherche contre la Sclérose en Plaques (ARSEP, France) and Schering France SA.

\section{Authors' affiliations}

M S A Deloire*, E Salort ${ }^{\star}, M$ Bonnet ${ }^{\star}$, M Boudineau, B Barroso, J-C Ouallet, E Galliaud, K G Petry, V Dousset, B Brochet, EA 2966 Neurobiology of Myelin Disorders Laboratory, University Victor Segalen, and Department of Neurology, CHU Bordeaux, France H Amieva, C Fabrigoule, INSERM U593, University Victor Segalen Y Arimone, Department of Pharmacology, University Victor Segalen C Pachai, Theralys Inc, Lyon, France

${ }^{*} M S A D, E S$, and MB made equal contributions to this work. Competing interests: none declared

\section{REFERENCES}

1 Mesulam MM. Large-scale neurocognitive networks and distributed processing for attention, language, and memory [review]. Ann Neurol 1990;28:597-613.

2 Rao SM, Leo GJ, Bernardin L, et al. Cognitive dysfunction in multiple sclerosis: frequency, patterns and prediction. Neurology 1991;41:685-91.

3 Ron MA, Callanan MM, Warrington EK. Cognitive abnormalities in multiple sclerosis: a psychometric and MRI study. Psychol Med. 1991;21: 59-68).

4 Heaton RK, Nelson LM, Thompson DS, et al. Neuropsychological findings in relapsing-remitting and chronic-progressive multiple sclerosis. J Consult Clin Psychol 1985;53:103-10.

5 Beatty WW, Goodkin DE, Monson N, et al. Anterograde and retrograde amnesia in patients with chronic progressive multiple sclerosis. Arch Neurol 1988;45:611-19

6 Feinstein A, Kartsounis LD, Miller DH, et al. Clinically isolated lesions of the type seen in multiple sclerosis: a cognitive, psychiatric, and MRI follow up study. J Neurol Neurosurg Psychiatry 1992;55:869-76.

7 Pelosi L, Geesken JM, Holly M, et al. Working memory impairment in early multiple sclerosis. Evidence from an event-related potential study of patients with clinically isolated myelopathy. Brain 1997;120:2039-58.

8 Achiron A, Barak Y. Cognitive impairment in probable multiple sclerosis. J Neurol Neurosurg Psychiatry 2003;74:443-6.

9 Grant I, McDonald WI, Trimble MR, et al. Deficient learning and memory in early and middle phases of multiple sclerosis. J Neurol Neurosurg Psychiatry 1984;47:250-5. 
10 Lyon-Caen 0 , Jouvent $\mathrm{R}$, Hauser $\mathrm{S}$, et al. Cognitive function in recent-onset demyelinating disease. Arch Neurol 1986;43:1138-41.

11 Amato MP, Ponziani G, Pracucci G, et al. Cognitive impairment in early-onset multiple sclerosis. Pattern, predictors, and impact on everyday life in a 4-year follow-up. Arch Neurol 1995;52:168-72.

12 Van den Burg W, Van Zomeren AH, Minderhoud JM, et al. Cognitive impairment in patients with multiple sclerosis and mild physical disability. Arch Neurol 1987;44:494-501

13 Zivadinov R, Sepcic J, Nasuelli D, et al. A longitudinal study of brain atrophy and cognitive disturbances in the early phase of relapsing-remitting multiple sclerosis. J Neurol Neurosurg Psychiatry 2001;70:773-80.

14 Zivadinov R, De Masi R, Nasuelli D, et al. MRI techniques and cognitive impairment in the early phase of relapsing-remitting multiple sclerosis. Neuroradiology 2001:43:272-8.

15 Comi G, Rovaris M, Leocani L, et al. Assessment of the damage of the cerebral hemispheres in MS using neuroimaging techniques [review]. J Neurol Sci 2000;172:S63-6.

16 Rao SM, Leo GJ, Haughton VM, et al. Correlation of magnetic resonance imaging with neuropsychological testing in multiple sclerosis. Neurology 1989:39:161-6.

17 Arnett PA, Rao SM, Bernardin L, et al. Relationship between frontal lobe lesions and Wisconsin Card Sorting Test performance in patients with multiple sclerosis. Neurology 1994;44:420-5.

18 Foong J, Rozewicz L, Quaghebeur G, et al. Executive function in multiple sclerosis. The role of frontal lobe pathology. Brain 1997;120:15-26.

19 Rovaris M, Filippi M, Falautano M, et al. Relation between MR abnormalities and patterns of cognitive impairment in multiple sclerosis. Neurology 1998;50:1601-8

20 Fulton JC, Grossman RI, Udupa J, et al. MR lesion load and cognitive function in patients with relapsing-remitting multiple sclerosis. Am J Neuroradiol 1999;20:1951-5.

21 Camp SJ, Stevenson VL, Thompson AJ, et al. Cognitive function in primary progressive and transitional progressive multiple sclerosis: a controlled study with MRI correlates. Brain 1999; 122:1341-8

22 Brochet B, Dousset V. Pathological correlates of magnetisation transfer imaging abnormalities in animal models and humans with multiple sclerosis [review]. Neurology 1999;53:S12-17.

23 Brochet B, Petry KG, Dousset V. Biology: the significance of MR parameters in multiple sclerosis. In: Tofts $P$, eds. Quantitative MRI of the brain: measuring change caused by disease. Chichester: John Wiley and Sons, 2003:477-501.

24 Rovaris M, Filippi M, Minicucci L, et al. Cortical/subcortical disease burden and cognitive impairment in patients with multiple sclerosis. Am J Neuroradiol 2000;21:402-8.

25 Filippi $M$, Tortorella $C$, Rovaris $M$, et al. Changes in the normal-appearing brain tissue and cognitive impairment in multiple sclerosis. J Neurol Neurosurg Psychiatry 2000;68:157-61.

26 Boringa JB, Lazeron RH, Reuling IE, et al. The brief repeatable battery of neuropsychological tests: normative values allow application in multiple sclerosis clinical practice. Multiple Sclerosis 2001;7:263-7.

27 Poser CM, Paty DW, Scheinberg L, et al. New diagnostic criteria for multiple sclerosis: guidelines for research protocols. Ann Neurol 1983;13:227-31.

28 Lublin FD, Reingold SC. Defining the clinical course of multiple sclerosis: results of an international survey. Neurology 1996;46:907-10.

29 Kurtzke JF. Rating neurologic impairment in multiple sclerosis: an expanded disability status scale (EDSS). Neurology 1983;33:1444-52.

30 Cutter GR, Baier ML, Rudick RA, et al. Development of a multiple sclerosis functional composite as a clinical trial outcome measure. Brain 1999; 122:871-82.

31 Fischer JS, Rudick RA, Cutter GR, et al. The Multiple Sclerosis Functional Composite Measure (MSFC): an integrated approach to MS clinical outcome assessment. National MS Society Clinical Outcomes Assessment Task Force [review]. Multiple Sclerosis 1999;5:244-50.

32 Kalkers NF, Bergers L, De Groot V, et al. Concurrent validity of the MS functional composite using MRI as biological disease marker. Neurology 2001;56:215-19.

33 Montgomery SA, Asberg M. A new depression scale designed to be sensitive to change. Br J Psychiatry 1979;134:382-9.

34 Sharrack B, Hughes RA. The Guy's Neurological Disability Scale (GNDS): a new disability measure for multiple sclerosis. Multiple Sclerosis 1999:5:223-33.

35 Snaith RP, Harrop FM, Newby DA, et al. Grade scores of the MontgomeryAsberg depression and the clinical anxiety scales. Br J Psychiatry, 1986;148, 599-601.

36 Rao SM. Cognitive Function Study Group, National Multiple Sclerosis society. A manual for the brief repeatable battery of neuropsychological tests in multiple sclerosis. New York: National Multiple Sclerosis Society, 1990

37 Amieva H, Lafont S, Auriacombe S, et al. Inhibitory breakdown and dementia of the Alzheimer type: a general phenomenon? J Clin Exp Neuropsychol 2002;24:503-16.
38 Bever CT, Grattan L, Panitch HS, et al. The brief repeatable battery of neuropsychological tests for multiple sclerosis: a preliminary serial study. Multiple Sclerosis 1995; 1:165-9.

39 Wechsler DA. Manual for the Wechsler adult intelligence scale-revised. New York: The Psychological Corporation, 1981.

40 Kaplan D, Goodglass A, Weintraub S. Boston naming test. Philadelphia: Lea and Febiger, 1983.

41 Ruff RM. Ruff figural fluency test professional manual. Odessa, FL: Psychological Assessment Resources Inc, 1988.

42 Vernay D, Gerbaud L, Clavelou P. Quality of life in multiple sclerosis. Rev Neurol (Paris), 2001;157:1139-42.

43 Rudick RA, Fisher E, Lee JC, et al. Use of the brain parenchymal fraction to measure whole brain atrophy in relapsing-remitting MS. Multiple Sclerosis Collaborative Research Group. Neurology 1999;53:1698-704.

44 Kalkers NF, Vrenken H, Uitdehaag BM, et al. Brain atrophy in multiple sclerosis: impact of lesions and of damage of whole brain tissue. Multiple Sclerosis 2002:8:410-14.

45 Dousset V, Grossman RI, Ramer KN, et al. Experimental allergic encephalomyelitis and multiple sclerosis: lesion characterization with magnetisation transfer imaging. Radiology 1992;183:483-91.

46 Bobholz JA, Rao SM. Cognitive dysfunction in multiple sclerosis: a review of recent developments [review]. Curr Opin Neurol 2003;16:283-8.

47 Solari A, Mancuso L, Motta A, et al. Comparison of two brief neuropsychological batteries in people with multiple sclerosis. Multiple Sclerosis 2002;8:169-76.

48 Sperling RA, Guttmann CR, Hohol MJ, et al. Regional magnetic resonance imaging lesion burden and cognitive function in multiple sclerosis: a longitudinal study. Arch Neurol 2001;58:115-21.

49 Vitkovitch $M$, Bishop S, Dancey $C$, et al. Stroop interference and negative priming in patients with multiple sclerosis. Neuropsychologia 2002;40:1570-6

50 Rao SM, Glatt S, Hammeke TA, et al. Chronic progressive multiple sclerosis. Relationship between cerebral ventricular size and neuropsychological impairment. Arch Neurol 1985;42:678-82.

51 Kujala P, Portin R, Ruutiainen J. The progress of cognitive decline in multiple sclerosis. A controlled 3-year follow-up. Brain 1997;120:289-97.

52 Laatu S, Hamalainen P, Revonsuo A, et al. Semantic memory deficit in multiple sclerosis; impaired understanding of conceptual meanings. J Neurol Sci 1999; 162:152-61.

53 Trapp BD, Peterson J, Ransohoff RM, et al. Axonal transection in the lesions of multiple sclerosis. N Engl J Med 1998;338:278-85.

54 Lazeron RH, Langdon DW, Filippi M, et al. Neuropsychological impairment in multiple sclerosis patients: the role of (juxta)cortical lesion on FLAIR. Multiple Sclerosis 2000;6:280-5

55 Chard DT, Griffin CM, Parker GJ, et al. Brain atrophy in clinically early relapsing-remitting multiple sclerosis. Brain 2002;125:327-37.

56 McGavern DB, Murray PD, Rivera-Quinones C, et al. Axonal loss results in spinal cord atrophy, electrophysiological abnormalities and neurological deficits following demyelination in a chronic inflammatory model of multiple sclerosis. Brain 2000;123:519-31.

57 Losseff NA, Wang L, Lai HM, et al. Progressive cerebral atrophy in multiple sclerosis. A serial MRI study. Brain 1996;119:2009-19.

58 Simon JH, Jacobs LD, Campion MK, et al. A longitudinal study of brain atrophy in relapsing multiple sclerosis. The Multiple Sclerosis Collaborative Research Group (MSCRG). Neurology 1999;53:139-48.

$59 \mathrm{Ge} \mathrm{Y,} \mathrm{Grossman} \mathrm{RI,} \mathrm{Udupa} \mathrm{JK,} \mathrm{et} \mathrm{al.} \mathrm{Brain} \mathrm{atrophy} \mathrm{in} \mathrm{relapsing-remitting}$ multiple sclerosis and secondary progressive multiple sclerosis: longitudinal quantitative analysis. Radiology 2000;214:665-70

60 Filippi M, Mastronardo G, Rocca MA, et al. Quantitative volumetric analysis of brain magnetic resonance imaging from patients with multiple sclerosis. J Neurol Sci 1998;158:148-53.

61 Liu C, Edwards S, Gong Q, et al. Three dimensional MRI estimates of brain and spinal cord atrophy in multiple sclerosis. I Neurol Neurosurg Psychiatry 1999;66:323-30

62 Fisher E, Rudick RA, Cutter G, et al. Relationship between brain atrophy and disability: an 8-year follow-up study of multiple sclerosis patients. Multiple Sclerosis 2000;6:373-7.

63 Benedict RH, Bakshi R, Simon JH, et al. Frontal cortex atrophy predicts cognitive impairment in multiple sclerosis. J Neuropsychiatry Clin Neurosci 2002;14:44-51.

64 Bermel RA, Bakshi R, Tjoa C, et al. Bicaudate ratio as a magnetic resonance imaging marker of brain atrophy in multiple sclerosis. Arch Neurol 2002;59:275-80.

65 Rovaris $M$, lannucci $G$, Falautano $M$, et al. Cognitive dysfunction in patients with mildly disabling relapsing-remitting multiple sclerosis: an exploratory study with diffusion tensor MR imaging. J Neurol Sci 2002;195:103-9. 